\title{
Social responsibility and work conditions : building a reference label, Démarche $T_{\circledast}$
}

\author{
Sylvain Biquand $d^{\mathrm{a}}$ and Benoit Zittel ${ }^{\mathrm{b}}$ \\ ${ }^{a}$ Abilis ergonomie, 10 rue Oberkampf, 75011, Paris, France \\ ${ }^{\mathrm{b}}$ Anthropie, 132 Boulevard Baille, 13005, Marseille, France
}

\begin{abstract}
Corporate Social Responsibility (CSR) is now considered in large and global companies and the recent publication of the ISO 26000 standard clarifies the targets. Based on our consultancy's experience for fifteen years in ergonomics mainly in French small and medium enterprises, we developed a label to coax and value efforts of companies in dealing with health and safety at the work place as required by ISO 26000 paragraph 6.4. The formal approach of ISO describes what should be achieved but gives no cue on how actual conditions of work should be improved. The label, called Démarche T (ie Process W where $\mathrm{W}$ stands for work) aims the management of work conditions as a process, giving visibility and credit to companies for their continuous involvement in the matter. We describe the items and processes that are part of our assessment. We first conduct an ergonomic diagnosis including the analysis of records on health, physical and psychological well-being, observations at the workplace and interviews with the workers. This diagnosis is followed by recommendations. The fulfillment of these is assessed yearly. Items under assessment include: - ergonomics, health and safety in the companies statements and their impact in actual project management; - relations with workers through the committee for health and safety; - actual results on health, safety and work conditions. On a local level, we give the companies passing the label a competitive edge in recruiting better candidates motivated by good work conditions, and help them fulfill ISO 26000 requirements, an increasingly decisive advantage to benefit from public regional and European support. Our paper describes the diagnosis and follow-up process.
\end{abstract}

Keywords: ISO 26000, ergonomics, work conditions, social responsibility

\section{Introduction}

Corporate Social Responsibility (CSR) is now considered in large and global companies and the recent publication of the ISO 26000 standard clarifies the targets. Based on our consultancy's experience for fifteen years in ergonomics mainly in French small and medium enterprises, we developed a label to coax and value efforts of companies in dealing with health and safety at the work place as required by ISO 26000 paragraph 6.4 . [1]. The formal approach of ISO describes what should be achieved but gives no cue on how actual conditions of work should be improved. The label, called Démarche $T \circledR$ ( $\mathrm{T}$ stands for travail ie work) aims the management of work conditions as a process, giving visibility and credit to companies for their continuous involvement in the matter. We describe the items and processes that are part of our assessment.

Démarche $\mathrm{T}$ is based on an initial diagnosis of work condition in the company following the methods of ergonomics intervention: observations and work analysis, documentary analysis, and interviews with workers on the workplace, the Human Resources management, the top and field production management and the hygiene and safety committee representing workers. After this initial diagnosis, a set of recommendation is submitted to the firm.

Through examples taken from our diagnosis in the company, we train the workers representatives and the direction in understanding physical and psychological work conditions and the leverage factors for improvement. The company management and operational staff are trained through action and project analysis to take charge of all aspects of work conditions.

Démarche $\mathrm{T}$ ( $\mathrm{T}$ process) helps enterprises to define and follow the management of work conditions. The predicaments are in line with ISO 26000 requirements on sustainable development including the preservation of the human resource.

\footnotetext{
* Corresponding authors e-mail: sbiquand@ergonome.com b.zittel@anthropie.net
} 
The $\mathrm{T}$ process integrates the crossed approaches of ergonomics, social psychology of work and organizations.

Objectives are

- to ensure the development of a methodology for the long term management of work conditions in line with the principles of ergonomics and prevention

- to provide a regular follow-up to each participating firm

- to promote and value the public image of the participating firm

- to prepare ground for the extended social responsibility system as per ISO 26000

Démarche $\mathrm{T}$ attests that the participating firms apply good practice as concerns work conditions, the conception and adaptation of work situations.

The labeled enterprise can communicate on its work conditions and valorize the integration of human development in its processes, respecting intelligence and integrity of the workers.

\section{Methods}

Our method aims at building an organization able to manage and improve current work conditions as well as to develop better projects.

The process organizes around three levels of responsibility in action. They are three organizational pillars for the development of an integrated method for improving work conditions (figure 1).

- a strategic commitment of the top management, gives the vision and frames the action

- the production services provide the analysis of work situations

- a steering committee is in charge of animation, synthesis and follow up

\subsection{First pillar: strategic commitment}

The top management is engaged and active to set work conditions as a fundamental and strategic asset. This aim shows up at different levels: communication, integration of unions and workers representatives, and formalized objectives

In its internal and public communication the firm shows a collective endeavor to:

- consider and improve work conditions

- ponder strategic options in relation to work conditions

- communicate on the management of work conditions

The firm also commits to integrate workers representatives to address work conditions:

- defines the roles of representatives as concerns work conditions
- creates a steering committee including workers' representation

The commitment for better work conditions is also reflected in management indicators to promote:

- indicators of work conditions

- annual objectives for work conditions

- contract objectives within each service

\subsection{Second pillar: assessment of work situations and projects}

Knowing work situations is necessary to improve work conditions. The company under Demarche $\mathrm{T}$ is able to trace work activity and its impact on worker's health and comfort. The firm commits to integrate the conditions of work in project development.

\subsubsection{Tasks and work situations}

The firm defines precisely the tasks given to each worker

- task description exists for each position. Task description is a tool to describe the fundamentals of a work situation and is a shared reference to formalize objectives.

The middle management commits to identify and regularly evaluate systemic and local constraints through analysis of work situations, leading to:

- the regular mapping of work situations and factors affecting workload

- the setting of indicators on work conditions

The principles of work activity ergonomics are the basis for the analysis of work situations including

- physiological and cognitive constraints

- organizational and psychosocial strain

The assessment tools are to be defined with the enterprise on the basis of real work situations. They will allow to map work constraints in the enterprise.

\subsubsection{Action and correction}

Production services will engage corrective actions on the basis of the map of work constraints and set priorities. Priorities are discussed in the steering committee on the basis of the level of physical and mental strain, technical feasibility, and the number of workers under constraints.

Local workgroup are set up including volunteer workers and a pilot. They follow a process including technical or organizational definition of the improvement, cost estimation, assessment of delays, results assessment and further adjustments if needed.

The consultants provide training to operational staff through project analysis, follow-up and validation. 


\subsection{Third pillar: coordination and debate}

The steering committee is a permanent structure debating work conditions and the coordination of improvement actions. It comprises, at least, of a member of the workers representation for health and safety (CHSCT), an executive member of the top management, an expert in work conditions (external consultant) and the medical doctor in charge of following the enterprise.

The steering committee draws a map of work conditions by deciding indicators that could be classified according to the level of physical or psychosocial strain. This classification allows priorities to be set and followed.

The steering committee debates and decides on:
- the regular synthesis of the map of work conditions and prioritization of actions

- the definition and follow-up of actions to improve work conditions

- the assessment of new projects development on work conditions

- the impacts of strategic orientations on future work conditions and the means to develop harmoniously work and business

The steering committee produces an annual report to be presented before the executive committee.

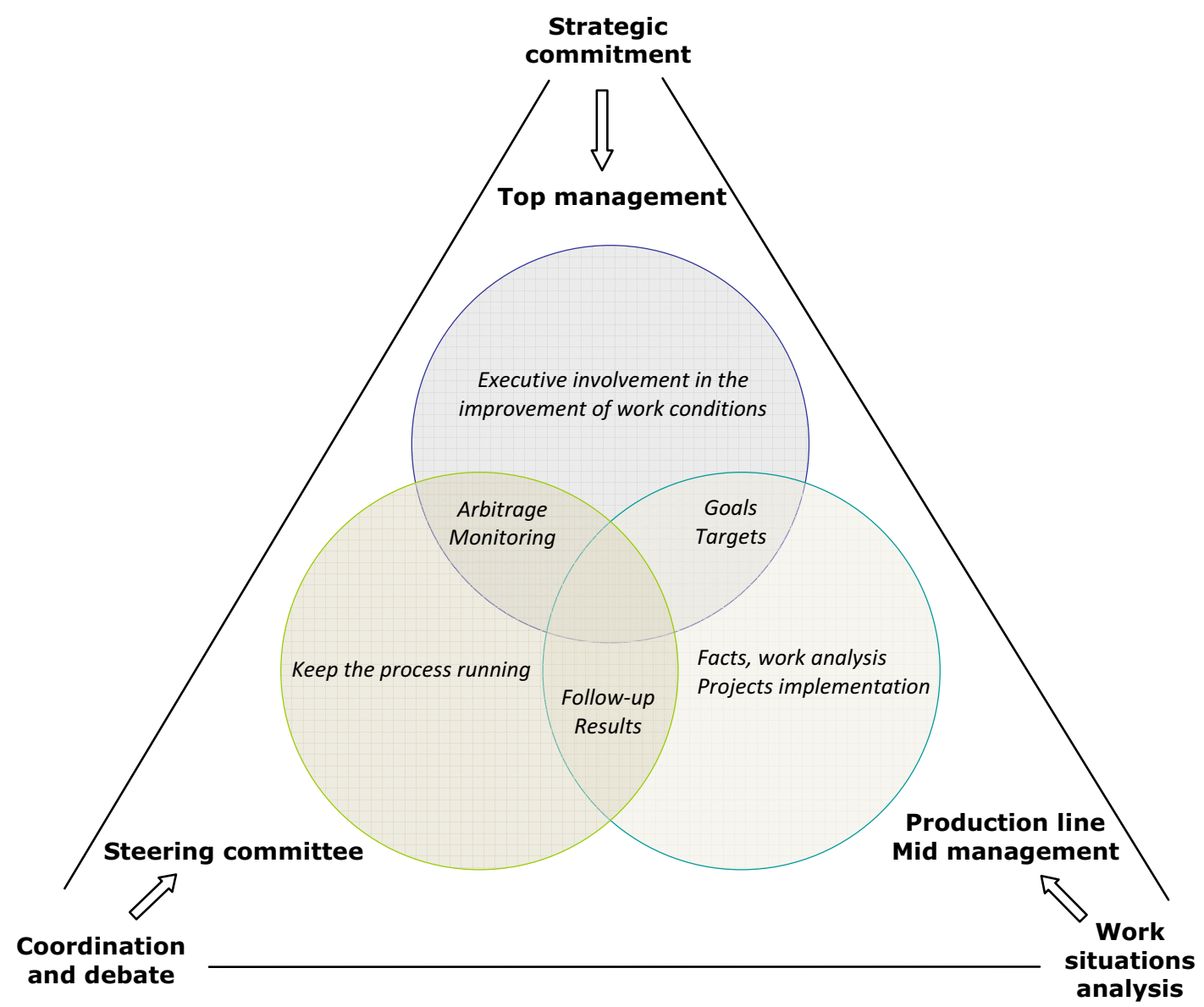

Figure 1 - The three pillars of Demarche T

\section{Discussion}

\subsection{ISO and Work conditions}

ISO 26000 addresses the whole area of Social Responsibility. The objective of social responsibility is to contribute to sustainable development which is a growing concern in society.

However, ISO 26000 is a guidance document, not a certification process; it provides a set of principles and objectives of social responsibility. ISO 26000 proposes each firm to define how they 
may apply these principles in their own organization.

According to ISO, results are to be expected on:

- improved reputation

- ability to attract and retain workers or members, customers, clients or users

- maintenance of employees' morale, commitment and productivity

- view of investors, owners, donors, sponsors and the financial community

- relationship with companies, governments, the media, suppliers, peers, customers and the community in which it operates.

Proposed as a guidance document, ISO 26000 does not prescribe a level of performance to be reached in each of the seven core subjects of social responsibility: Community involvement and development, Human rights, Environment, Consumer issues, Fair operating practices, Labor practices and Organizational Governance.

Through our action on work conditions, we aim at topics labeled Health and Safety at work (6.4.6), in the chapter concerning Labor Practices (6.4). However, the comprehensive approach of work conditions proposed with Demarche $\mathrm{T}$, also links with governance and community involvement.

\subsection{Results and processes}

Demarche $\mathrm{T}$ engages the firm in a continuous process and integrating the label follows three steps:

- the diagnosis of work situations and management processes as regards work conditions. The diagnosis results in setting up the program for action.

- the first assessment of actions at year+1 and certificate delivery.

- the annual audit of results and processes

After the initial diagnosis, the recommendations comprises both the identification of physical improvements to be implemented in work situations, and improved rules for project development including the assessment of future work situations and their impact on workers activity and work conditions.
Improved work conditions are assessed through direct observation and interviews in work situations. Indirect results are also known to reflect work strain as the figures of absenteeism, occupational diseases and accidents.

Improved processes of conception are also expected. Our assistance to the steering committee and to operational management teams aims to provide tools as well as organizational training to integrate work activity as a factor of project development. The development of new projects should integrate the anticipation of future work situations, and the assessment of their impact on work conditions.

The targets of Demarche $\mathrm{T}$ are small and medium enterprises where it is important to develop structures and processes to improve work conditions. Our methodology, demarche T, deepens the proposals of ISO by considering and monitoring results on health at work and by setting processes that will create a better workplace as well as a better involvement of workers.

\section{Reference}

[1] ISO 26000:2010 - International Standards OrganizationGeneva. 